\title{
Synthesis and Evaluation of the Acetylcholinesterase Inhibitory Activities of Some Flavonoids Derived from Naringenin
}

\author{
The-Huan Tran (iD, Thi-Thu-Hien Vo $(\mathbb{D}$, Thi-Quynh-Nhi Vo $\mathbb{D}$, Thi-Cam-Nhung Cao $(\mathbb{D}$, \\ and Thai-Son Tran $\mathbb{D}$
}

\begin{abstract}
Department of Pharmaceutical Chemistry, Faculty of Pharmacy, University of Medicine and Pharmacy, Hue University, Hue 530000, Vietnam
\end{abstract}

Correspondence should be addressed to Thai-Son Tran; tthaison@hueuni.edu.vn

Received 16 August 2021; Revised 3 November 2021; Accepted 9 November 2021; Published 30 November 2021

Academic Editor: Tullio Florio

\begin{abstract}
Copyright (c) 2021 The-Huan Tran et al. This is an open access article distributed under the Creative Commons Attribution License, which permits unrestricted use, distribution, and reproduction in any medium, provided the original work is properly cited.
\end{abstract}

\begin{abstract}
Alzheimer's disease (AD) is an irreversible neurodegenerative disease that affects many older people adversely. AD has been putting a huge socioeconomic burden on the healthcare systems of many developed countries with aging populations. The need for new therapies that can halt or reverse the progression of the disease is now extremely great. A research approach in the finding new treatment for $\mathrm{AD}$ that has attracted much interest from scientists for a long time is the reestablishment of cholinergic transmission through inhibition of acetylcholinesterase (AChE). Naringenin is a flavonoid with the potential inhibitory activity against AChE. From naringenin, many other flavonoid derivatives, such as flavanones and chalcones, can be synthesized. In this study, by applying the Williamson method, nine flavonoid derivatives were synthesized, including four flavanones and five chalcones. The evaluation of AChE inhibitory activity by the Ellman method showed that there were four substances $(\mathbf{2}, \mathbf{4 , 5}$, and 7) with relatively good biological activities $\left(\mathrm{IC}_{50}<100 \mu \mathrm{M}\right.$ ), and these biological activities were better than that of naringenin. The molecular docking revealed that strong interactions with amino acid residue Ser200 of the catalytic triad and those of the peripheral region of the enzyme were crucial for strong effects against AChE. Compound 7 had the strongest AChE inhibitory activity $\left(\mathrm{IC}_{50} 13.0 \pm 1.9 \mu \mathrm{M}\right)$. This substance could be used for further studies.
\end{abstract}

\section{Introduction}

Alzheimer's disease (AD) is one of the greatest medical challenges facing us in the current era. This is the most common form of dementia. The disease is manifested by intellectual impairment, memory loss, language disorders, and the disability in movements as well as cognition, leading to serious impacts on occupational activities and social communication of the patients. $\mathrm{AD}$ also has a heavy impact on the patient's family and people around because caring for an $\mathrm{AD}$ patient is often difficult and expensive. AD worsens over time and eventually leads to death [1]. Worldwide, the number of people living with AD-related dementia is around 50 million (2018), and this figure is expected to grow to 152 million by 2050 [2], with trillions of US dollars paid for the global healthcare for the disease [3].
The cholinergic hypothesis suggests that cholinergic neurotransmission is likely to play a vital role in memory, learning, concentration, and other advanced neural functions. Several studies have suggested additional roles of the cholinergic system in overall brain homeostasis and neural plasticity. Thus, the cholinergic system has occupied a central place in many research studies related to normal cognitive function and age-related dementia, including $\mathrm{AD}$ [4]. Acetylcholinesterase (AChE, EC 3.1.1.7) catalyzes the hydrolysis of acetylcholine and some other neurotransmitter choline esters. $\mathrm{AChE}$ is found primarily at cholinergic synapses, where it rapidly degrades acetylcholine to release choline and acetate. Hence, AChE plays an essential role in cholinergic neurotransmission. Acetylcholinesterase inhibitors increase the levels of acetylcholine in synaptic clefts and are one of the very few proven clinically effective therapies in 
the treatment of AD-induced dementia. Therefore, AChE is an important therapeutic target for the disease currently [5-8].

Flavonoid is a large group of naturally occurring compounds with diverse biological activities [9]. Naringenin is a natural flavonoid found in many plants [10]. As a flavonoid, this compound has many biological activities such as antioxidant [11], anti-inflammatory [12], antiviral [13], and anticancer [14]. In particular, naringenin has been reported to have AChE inhibitory activity [15].

In this study, flavonoid derivatives were synthesized from naringenin and were evaluated for their inhibitory effects on AChE. The mode of interaction to the enzyme target of these compounds and their predicted profiles in physicochemical properties, pharmacokinetics, and toxicities were also investigated to serve as premises for further studies.

\section{Experimental}

2.1. Materials and Methods. All chemicals were obtained from commercial suppliers and used without further purification. Melting points determination, UV (ultraviolet), IR (infrared), HR-MS (high-resolution mass spectrometry), ${ }^{1} \mathrm{H}-\mathrm{NMR}$ (proton nuclear magnetic resonance), and ${ }^{13} \mathrm{C}$ NMR (carbon-13 nuclear magnetic resonance) spectra elucidation and all computation processes were performed on the systems as described earlier [16].

\subsection{Synthesis of Flavonoid Derivatives from Naringenin.} The Williamson method [17] was applied for the synthesis of flavonoid derivatives from naringenin. The reactions are indicated in Scheme 1 and are described briefly as follows: naringenin was dissolved in acetone. $\mathrm{K}_{2} \mathrm{CO}_{3}$ and dialkyl sulfate or alkyl bromide were then added. The reaction mixture was heated at $40-45^{\circ} \mathrm{C}$ and was monitored by thin layer chromatography with the appropriate solvent systems. At the end of the reaction, the mixture was filtered to remove the insoluble solid. The obtained solution was evaporated under reduced pressure. The crude product was purified by recrystallization or by column chromatography with the appropriate solvent systems. The structures of the synthetic substances were elucidated by UV, IR, HR-MS, ${ }^{1} \mathrm{H}-\mathrm{NMR}$, and ${ }^{13} \mathrm{C}-\mathrm{NMR}$ spectra.

2.2.1. 4',5-Dihydroxy-7-methoxyflavanone (1). White, needle-shaped crystals slightly soluble in water, soluble in methanol, highly soluble in acetone, and sparingly soluble in dichloromethane. Yield is $54 \%$. Melting point: $106-107^{\circ} \mathrm{C}$. $\mathrm{UV}\left(\lambda_{\max } \mathrm{nm}, \mathrm{MeOH}\right): 287 . \mathrm{IR}\left(\nu \mathrm{cm}^{-1}, \mathrm{KBr}\right): 3523\left(\nu_{\mathrm{OH}}\right)$, $3115\left(\nu_{\mathrm{C}-\mathrm{H} \mathrm{sp} 2}\right), 2893\left(\nu_{\mathrm{C}-\mathrm{H} \mathrm{sp} 3}\right), 1640\left(\nu_{\mathrm{C}=\mathrm{O}}\right), 1618\left(\nu_{\mathrm{C}=\mathrm{C}}\right)$, $1444\left(\nu_{\mathrm{C}=\mathrm{C}}\right)$, and $1153\left(\nu_{\mathrm{C}-\mathrm{O}}\right)$. HR-MS (ESI) m/z: $[\mathrm{M}+\mathrm{H}]^{+}$ calculated for $\mathrm{C}_{16} \mathrm{H}_{15} \mathrm{O}_{5}$ 287.0919; found 287.0911. ${ }^{1} \mathrm{H}-\mathrm{NMR}$ $\left(600 \mathrm{MHz}, \mathrm{DMSO}-d_{6}\right): \delta 12.11(\mathrm{~s}, 1 \mathrm{H}, \mathrm{OH}) ; 9.58(\mathrm{~s}, 1 \mathrm{H}, \mathrm{OH})$; 7.33-7.31 (m, 2H, $\left.\mathrm{H}_{\mathrm{Ar}}\right) ; 6.80-6.78\left(\mathrm{~m}, 2 \mathrm{H}, \mathrm{H}_{\mathrm{Ar}}\right) ; 6.10(\mathrm{~d}$, $\left.J=2.4 \mathrm{~Hz}, 1 \mathrm{H}, \mathrm{H}_{\mathrm{Ar}}\right) ; 6.08\left(\mathrm{~d}, J=2.4 \mathrm{~Hz}, 1 \mathrm{H}, \mathrm{H}_{\mathrm{Ar}}\right) ; 5.49$ (dd, $J=12.6 ; 3.0 \mathrm{~Hz}, 1 \mathrm{H}, \mathrm{O}-\mathrm{CH}<) ; 3.78\left(\mathrm{~s}, 3 \mathrm{H}, \mathrm{CH}_{3}\right) ; 3.32(\mathrm{dd}$, $\left.J=17.4 ; 12.6 \mathrm{~Hz}, 1 \mathrm{H}, \mathrm{CH}_{2}\right)$; and $2.72(\mathrm{dd}, J=17.4 ; 3.0 \mathrm{~Hz}, 1 \mathrm{H}$,
$\left.\mathrm{CH}_{2}\right) .{ }^{13} \mathrm{C}-\mathrm{NMR}\left(150 \mathrm{MHz}, \mathrm{DMSO}-d_{6}\right): \delta 196.9(\mathrm{C}=\mathrm{O})$, $167.4,163.1,162.8,157.7,128.6,128.3,115.1,102.5,94.6$, 93.7, 78.6 $(\mathrm{O}-\mathrm{CH}<), 55.8\left(\mathrm{CH}_{3}\right)$, and $42.0\left(\mathrm{CH}_{2}\right)$.

2.2.2. 5-Hydroxy-4',7-dimethoxyflavanone (2). White, needle-shaped crystals insoluble in water, sparingly soluble in methanol, soluble in acetone, and highly soluble in dichloromethane. Yield is $68 \%$. Melting point: $116-117^{\circ} \mathrm{C}$. $\mathrm{UV}\left(\lambda_{\max } \mathrm{nm}, \mathrm{MeOH}\right): 288$. IR $\left(\nu \mathrm{cm}^{-1}, \mathrm{KBr}\right): 3446\left(\nu_{\mathrm{OH}}\right)$, $3093\left(\nu_{\mathrm{C}-\mathrm{H} \text { sp2 }}\right), 2914\left(\nu_{\mathrm{C}-\mathrm{H} \mathrm{sp} 3}\right), 1631\left(\nu_{\mathrm{C}=\mathrm{O}}\right), 1579\left(\nu_{\mathrm{C}=\mathrm{C}}\right)$, $1433\left(\nu_{\mathrm{C}=\mathrm{C}}\right)$, and $1157\left(\nu_{\mathrm{C}-\mathrm{O}}\right)$. HR-MS (ESI) m/z: $[\mathrm{M}+\mathrm{H}]^{+}$ calculated for $\mathrm{C}_{17} \mathrm{H}_{17} \mathrm{O}_{5}$ 301.1076; found 301.1066. ${ }^{1} \mathrm{H}-\mathrm{NMR}$ $\left(500 \mathrm{MHz}, \mathrm{DMSO}-d_{6}\right): \delta 12.10(\mathrm{~s}, 1 \mathrm{H}, \mathrm{OH}) ; 7.46-7.43(\mathrm{~m}$, $\left.2 \mathrm{H}, \mathrm{H}_{\mathrm{Ar}}\right) ; 6.99-6.96\left(\mathrm{~m}, 2 \mathrm{H}, \mathrm{H}_{\mathrm{Ar}}\right) ; 6.12(\mathrm{~d}, J=2.0 \mathrm{~Hz}, 1 \mathrm{H}$, $\left.\mathrm{H}_{\mathrm{Ar}}\right) ; 6.08\left(\mathrm{~d}, J=2.0 \mathrm{~Hz}, 1 \mathrm{H}, \mathrm{H}_{\mathrm{Ar}}\right) ; 5.55(\mathrm{dd}, J=13.0 ; 3.0 \mathrm{~Hz}$, $1 \mathrm{H}, \mathrm{O}-\mathrm{CH}<) ; 3.79$ (s, 3H, $\left.\mathrm{CH}_{3}\right) ; 3.77\left(\mathrm{~s}, 3 \mathrm{H}, \mathrm{CH}_{3}\right) ; 3.33$ (dd, $\left.J=17.0 ; 13.0 \mathrm{~Hz}, 1 \mathrm{H}, \mathrm{CH}_{2}\right)$; and $2.77(\mathrm{dd}, \mathrm{J}=17.0 ; 3.0 \mathrm{~Hz}, 1 \mathrm{H}$, $\left.\mathrm{CH}_{2}\right) .{ }^{13} \mathrm{C}-\mathrm{NMR}\left(125 \mathrm{MHz}, \mathrm{DMSO}-d_{6}\right): \delta 196.7(\mathrm{C}=\mathrm{O})$, $167.4,163.1,162.7,159.4,130.4,128.2,113.8,102.5,94.6$, 93.7, $78.3(\mathrm{O}-\mathrm{CH}<), 55.8\left(\mathrm{CH}_{3}\right), 55.1\left(\mathrm{CH}_{3}\right)$, and $42.0\left(\mathrm{CH}_{2}\right)$.

2.2.3. (E)-2',4,4', $6^{\prime}$-Tetramethoxychalcone (3). Green, needle-shaped crystals insoluble in water, sparingly soluble in methanol, soluble in acetone, and highly soluble in dichloromethane. Yield is $47 \%$. Melting point: $123-124^{\circ} \mathrm{C}$. $\mathrm{UV}\left(\lambda_{\max } \mathrm{nm}, \mathrm{MeOH}\right): 327 . \mathrm{IR}\left(\nu \mathrm{cm}^{-1}, \mathrm{KBr}\right): 3008\left(\nu_{\mathrm{C}-\mathrm{H}}\right.$ $\left.{ }_{\mathrm{sp} 2}\right), 2937\left(\nu_{\mathrm{C}-\mathrm{H} \mathrm{sp} 3}\right), 1672\left(\nu_{\mathrm{C}=\mathrm{O}}\right), 1600\left(\nu_{\mathrm{C}=\mathrm{C}}\right), 1465\left(\nu_{\mathrm{C}=\mathrm{C}}\right)$, and $1126\left(\nu_{\mathrm{C}-\mathrm{O}}\right)$. HR-MS (ESI) $\mathrm{m} / \mathrm{z}:[\mathrm{M}+\mathrm{H}]^{+}$calculated for $\mathrm{C}_{19} \mathrm{H}_{21} \mathrm{O}_{5}$ 329.1389; found 329.1389. ${ }^{1} \mathrm{H}-\mathrm{NMR}(500 \mathrm{MHz}$, DMSO- $\left.d_{6}\right): \delta 7.61-7.59\left(\mathrm{~m}, 2 \mathrm{H}, \mathrm{H}_{\mathrm{Ar}}\right) ; 7.18(\mathrm{~d}, J=16.0 \mathrm{~Hz}$, $\left.1 \mathrm{H}, \mathrm{H}_{\beta}\right) ; 6.95-6.94\left(\mathrm{~m}, 2 \mathrm{H}, \mathrm{H}_{\mathrm{Ar}}\right) ; 6.83(\mathrm{~d}, J=16.0 \mathrm{~Hz}, 1 \mathrm{H}$, $\mathrm{H}_{\alpha}$ ); 6.31 (s, 2H, $\left.\mathrm{H}_{\mathrm{Ar}}\right) ; 3.83\left(\mathrm{~s}, 3 \mathrm{H}, \mathrm{CH}_{3}\right) ; 3.78$ (s, 3H, $\mathrm{CH}_{3}$ ); and $3.71\left(\mathrm{~s}, 6 \mathrm{H}, \mathrm{CH}_{3}\right) .{ }^{13} \mathrm{C}-\mathrm{NMR}\left(125 \mathrm{MHz}, \mathrm{DMSO}-d_{6}\right): \delta$ $193.2(\mathrm{C}=\mathrm{O}), 161.7,161.1,157.9,143.6,130.2,126.9,126.8$, 114.4, 111.2, 91.0, $55.7\left(\mathrm{CH}_{3}\right), 55.3\left(\mathrm{CH}_{3}\right)$, and $55.2\left(\mathrm{CH}_{3}\right)$.

2.2.4. (E)-2',4,4'-Triethoxy-6'-hydroxychalcone (4). Yellow, needle-shaped crystals insoluble in water, slightly soluble in methanol, soluble in acetone, and highly soluble in dichloromethane. Yield is $52 \%$. Melting point: $135-136^{\circ} \mathrm{C}$. $\mathrm{UV}\left(\lambda_{\max } \mathrm{nm}, \mathrm{MeOH}\right): 364 . \mathrm{IR}\left(\nu \mathrm{cm}^{-1}, \mathrm{KBr}\right): 3446\left(\nu_{\mathrm{OH}}\right)$, $2981\left(\nu_{\mathrm{C}-\mathrm{H} \mathrm{sp} 2}\right), 2933\left(\nu_{\mathrm{C}-\mathrm{H} \mathrm{sp} 3}\right), 1622\left(\nu_{\mathrm{C}=\mathrm{O}}\right), 1554\left(\nu_{\mathrm{C}=\mathrm{C}}\right)$, $1473\left(\nu_{\mathrm{C}=\mathrm{C}}\right)$, and $1118\left(\nu_{\mathrm{C}-\mathrm{O}}\right)$. HR-MS (ESI) m/z: $[\mathrm{M}+\mathrm{H}]^{+}$ calculated for $\mathrm{C}_{21} \mathrm{H}_{25} \mathrm{O}_{5}$ 357.1702; found 357.1702. ${ }^{1} \mathrm{H}-\mathrm{NMR}$ $\left(500 \mathrm{MHz}, \mathrm{DMSO}-d_{6}\right): \delta 13.71(\mathrm{~s}, 1 \mathrm{H}, \mathrm{OH}) ; 7.78(\mathrm{~d}$, $\left.J=15.5 \mathrm{~Hz}, 1 \mathrm{H}, \mathrm{H}_{\beta}\right) ; 7.63\left(\mathrm{~d}, J=15.5 \mathrm{~Hz}, 1 \mathrm{H}, \mathrm{H}_{\alpha}\right) ; 7.61-7.59$ $\left(\mathrm{m}, 2 \mathrm{H}, \mathrm{H}_{\mathrm{Ar}}\right) ; 6.99-6.97\left(\mathrm{~m}, 2 \mathrm{H}, \mathrm{H}_{\mathrm{Ar}}\right) ; 6.08(\mathrm{~d}, J=2.0 \mathrm{~Hz}$, $\left.\mathrm{H}_{\mathrm{Ar}}\right) ; 6.06\left(\mathrm{~d}, J=2.0 \mathrm{~Hz}, \mathrm{H}_{\mathrm{Ar}}\right) ; 4.16-4.07\left(\mathrm{~m}, 6 \mathrm{H}, \mathrm{CH}_{2}\right) ; 1.42$ $\left(\mathrm{t}, J=7.0 \mathrm{~Hz}, 3 \mathrm{H}, \mathrm{CH}_{3}\right) ; 1.34\left(\mathrm{t}, J=7.0 \mathrm{~Hz}, 3 \mathrm{H}, \mathrm{CH}_{3}\right)$; and $1.33\left(\mathrm{t}, \mathrm{J}=7.0 \mathrm{~Hz}, 3 \mathrm{H}, \mathrm{CH}_{3}\right) .{ }^{13} \mathrm{C}-\mathrm{NMR}(125 \mathrm{MHz}, \mathrm{DMSO}-$ $\left.d_{6}\right): \delta 191.8(\mathrm{C}=\mathrm{O}), 165.9,164.6,161.1,160.2,141.5,129.6$, 127.2 , 124.8, 114.7, 105.9, 94.1, 91.7, $64.2\left(\mathrm{CH}_{2}\right), 63.3\left(\mathrm{CH}_{2}\right)$, $63.0\left(\mathrm{CH}_{2}\right), 14.1\left(\mathrm{CH}_{3}\right)$, and $13.9\left(\mathrm{CH}_{3}\right)$.

2.2.5. 7-Allyloxy-4',5-dihydroxyflavanone (5). Yellow powder and insoluble in water, sparingly soluble in methanol and in dichloromethane, and highly soluble in acetone. Yield 
is $58 \%$. Melting point: $103-104^{\circ} \mathrm{C} \cdot U V\left(\lambda_{\max } \mathrm{nm}, \mathrm{MeOH}\right): 288$. IR $\left(\nu \mathrm{cm}^{-1}, \mathrm{KBr}\right): 3406\left(\nu_{\mathrm{OH}}\right), 3024\left(\nu_{\mathrm{C}-\mathrm{H} \mathrm{sp} 2}\right), 2918\left(\nu_{\mathrm{C}-\mathrm{H} \mathrm{sp} 3}\right)$, $1635\left(v_{\mathrm{C}=\mathrm{O}}\right), 1570\left(v_{\mathrm{C}=\mathrm{C}}\right), 1446\left(v_{\mathrm{C}=\mathrm{C}}\right)$, and $1166\left(v_{\mathrm{C}-\mathrm{O}}\right)$. HRMS (ESI) m/z: $[\mathrm{M}+\mathrm{H}]^{+}$calculated for $\mathrm{C}_{18} \mathrm{H}_{17} \mathrm{O}_{5} 313.1076$; found 313.1076. ${ }^{1} \mathrm{H}-\mathrm{NMR}\left(600 \mathrm{MHz}, \mathrm{DMSO}-d_{6}\right): \delta 12.09$ (s, $1 \mathrm{H}, \mathrm{OH}) ; 9.59(\mathrm{~s}, 1 \mathrm{H}, \mathrm{OH}) ; 7.32\left(\mathrm{~d}, J=8.4 \mathrm{~Hz}, 2 \mathrm{H}, \mathrm{H}_{\mathrm{Ar}}\right) ; 6.79$ $\left(\mathrm{d}, \mathrm{J}=8.4 \mathrm{~Hz}, 2 \mathrm{H}, \mathrm{H}_{\mathrm{Ar}}\right) ; 6.11\left(\mathrm{~d}, J=2.4 \mathrm{~Hz}, 1 \mathrm{H}, \mathrm{H}_{\mathrm{Ar}}\right) ; 6.09$ (d, $\left.J=2.4 \mathrm{~Hz}, 1 \mathrm{H}, \mathrm{H}_{\mathrm{Ar}}\right) ; 6.01-5.97(\mathrm{~m}, 1 \mathrm{H}, \mathrm{CH}=) ; 5.48(\mathrm{dd}$, $J==12.6 ; 3.0 \mathrm{~Hz}, 1 \mathrm{H}, \mathrm{O}-\mathrm{CH}<) ; 5.37(\mathrm{dd}, J==17.4 ; 1.8 \mathrm{~Hz}$, $\left.1 \mathrm{H}, \mathrm{CH}_{2}=\right) ; 5.26\left(\mathrm{dd}, J=10.8 ; 1.8 \mathrm{~Hz}, 1 \mathrm{H}, \mathrm{CH}_{2}=\right) ; 4.62(\mathrm{~d}$, $\left.J=5.4 \mathrm{~Hz}, 2 \mathrm{H}, \mathrm{O}-\mathrm{CH}_{2}-\right) ; 3.32(\mathrm{dd}, \mathrm{J}=17.4 ; 12.6 \mathrm{~Hz}, 1 \mathrm{H}$, $\left.-\mathrm{CH}_{2-}\right)$; and $2.71\left(\mathrm{dd}, J=17.4 ; 3.0 \mathrm{~Hz}, 1 \mathrm{H},-\mathrm{CH}_{2}-\right) .{ }^{13} \mathrm{C}-\mathrm{NMR}$ $\left(150 \mathrm{MHz}, \mathrm{DMSO}-d_{6}\right): \delta 196.9(\mathrm{C}=\mathrm{O}), 166.2,163.1,162.8$, $157.7,132.8,128.6,128.3,118.0,115.1,102.6,95.2,94.3,78.6$ $(\mathrm{O}-\mathrm{CH}<), 68.7\left(\mathrm{O}-\mathrm{CH}_{2}\right)$, and $42.0\left(\mathrm{CH}_{2}\right)$.

2.2.6. 4',7-Diallyloxy-5-hydroxyflavanone (6). Yellow powder and insoluble in water, sparingly soluble in methanol, soluble in acetone, and highly soluble in dichloromethane. Yield is $61 \%$. Melting point: $61-62^{\circ} \mathrm{C}$. UV $\left(\lambda_{\max } \mathrm{nm}, \mathrm{MeOH}\right)$ : 288. IR $\left(\nu \mathrm{cm}^{-1}, \mathrm{KBr}\right): 3446\left(v_{\mathrm{OH}}\right), 3080\left(v_{\mathrm{C}-\mathrm{H} \mathrm{sp} 2}\right), 2916\left(v_{\mathrm{C}-\mathrm{H}}\right.$ sp3 $), 1651\left(\nu_{\mathrm{C}=\mathrm{O}}\right), 1616\left(\nu_{\mathrm{C}=\mathrm{C}}\right), 1514\left(\nu_{\mathrm{C}=\mathrm{C}}\right)$, and $1170\left(\nu_{\mathrm{C}-\mathrm{O}}\right)$. HR-MS (ESI) m/z: $[\mathrm{M}+\mathrm{H}]^{+}$calculated for $\mathrm{C}_{21} \mathrm{H}_{21} \mathrm{O}_{5} 353.1389$; found 353.1388. ${ }^{1} \mathrm{H}-\mathrm{NMR}\left(600 \mathrm{MHz}, \mathrm{DMSO}-d_{6}\right): \delta 12.08$ (s, $1 \mathrm{H}, \mathrm{OH}) ; 7.44\left(\mathrm{~d}, J=8.4 \mathrm{~Hz}, 2 \mathrm{H}, \mathrm{H}_{\mathrm{Ar}}\right) ; 7.00(\mathrm{~d}, J=8.4 \mathrm{~Hz}, 2 \mathrm{H}$, $\left.\mathrm{H}_{\mathrm{Ar}}\right) ; 6.13\left(\mathrm{~d}, J=2.4 \mathrm{~Hz}, 1 \mathrm{H}, \mathrm{H}_{\mathrm{Ar}}\right) ; 6.10\left(\mathrm{~d}, J=2.4 \mathrm{~Hz}, 1 \mathrm{H}, \mathrm{H}_{\mathrm{Ar}}\right)$; 6.07-5.96 (m, 2H, CH=); $5.55(\mathrm{dd}, \mathrm{J}=12.6 ; 3.0 \mathrm{~Hz}, 1 \mathrm{H}$, $\mathrm{O}-\mathrm{CH}<)$; 5.41-5.36 (m, $\left.2 \mathrm{H}, \mathrm{CH}_{2}=\right)$; 5.27-5.25 (m, $\left.2 \mathrm{H}, \mathrm{CH}_{2}=\right)$; $4.63\left(\mathrm{~d}, J=5.4 \mathrm{~Hz}, 2 \mathrm{H}, \mathrm{O}-\mathrm{CH}_{2}-\right) ; 4.58(\mathrm{~d}, J=5.4 \mathrm{~Hz}, 2 \mathrm{H}$, $\mathrm{O}-\mathrm{CH} 2-$ ); 3.34 (dd, $\left.J=17.4 ; 12.6 \mathrm{~Hz}, 1 \mathrm{H}, \mathrm{CH}_{2}\right)$; and 2.76 (dd, $\left.J=17.4 ; 3.0 \mathrm{~Hz}, 1 \mathrm{H}, \mathrm{CH}_{2}\right) .{ }^{13} \mathrm{C}-\mathrm{NMR}\left(150 \mathrm{MHz}, \mathrm{DMSO}-d_{6}\right): \delta$ $196.7(\mathrm{C}=\mathrm{O}), 166.2,163.1,162.7,158.3,133.5,132.8,130.5$, $128.2,118.0,117.4,114.6,102.6,95.2,94.3,78.3(\mathrm{O}-\mathrm{CH}<), 68.7$ $\left(\mathrm{O}-\mathrm{CH}_{2}\right), 68.1\left(\mathrm{O}-\mathrm{CH}_{2}\right)$, and $41.9\left(\mathrm{CH}_{2}\right)$.

\subsection{7. (E)-2' $4,4^{\prime}$-Triallyloxy-6' -hydroxychalcone}

(7).

Yellow, needle-shaped crystals insoluble in water, sparingly soluble in methanol, soluble in acetone, and highly soluble in dichloromethane. Yield is $66 \%$. Melting point: $72-73^{\circ} \mathrm{C}$. UV $\left(\lambda_{\max } \mathrm{nm}, \mathrm{MeOH}\right): 364$ IR $\left(\nu \mathrm{cm}^{-1}, \mathrm{KBr}\right): 3452\left(\nu_{\mathrm{OH}}\right), 3080$ $\left(\nu_{\mathrm{C}-\mathrm{H} \mathrm{sp} 2}\right), 2933\left(v_{\mathrm{C}-\mathrm{H} \mathrm{sp} 3}\right), 1627\left(\nu_{\mathrm{C}=\mathrm{O}}\right), 1554\left(v_{\mathrm{C}=\mathrm{C}}\right), 1423$ $\left(\nu_{\mathrm{C}=\mathrm{C}}\right)$, and $1166\left(\nu_{\mathrm{C}-\mathrm{O}}\right)$. HR-MS (ESI) $\mathrm{m} / \mathrm{z}:[\mathrm{M}+\mathrm{H}]^{+}$calculated for $\mathrm{C}_{24} \mathrm{H}_{25} \mathrm{O}_{5}$ 393.1702; found 393.1707. ${ }^{1} \mathrm{H}-\mathrm{NMR}$ $\left(500 \mathrm{MHz}, \mathrm{DMSO}-d_{6}\right): \delta 13.60(\mathrm{~s}, 1 \mathrm{H}, \mathrm{OH}) ; 7.73(\mathrm{~d}, J=15.5 \mathrm{~Hz}$, $\left.1 \mathrm{H}, \mathrm{H}_{\beta}\right) ; 7.64\left(\mathrm{~d}, J=15.5 \mathrm{~Hz}, 1 \mathrm{H}, \mathrm{H}_{\alpha}\right) ; 7.61(\mathrm{~d}, J=9.0 \mathrm{~Hz}, 2 \mathrm{H}$, $\left.\mathrm{H}_{\mathrm{Ar}}\right) ; 7.00\left(\mathrm{~d}, J=9.0 \mathrm{~Hz}, 2 \mathrm{H}, \mathrm{H}_{\mathrm{Ar}}\right) ; 6.12\left(\mathrm{~d}, J=2.5 \mathrm{~Hz}, 1 \mathrm{H}, \mathrm{H}_{\mathrm{Ar}}\right)$; $6.17\left(\mathrm{~d}, J=2.5 \mathrm{~Hz}, 1 \mathrm{H}, \mathrm{H}_{\mathrm{Ar}}\right) ; 6.14-5.99(\mathrm{~m}, 3 \mathrm{H}, \mathrm{O}-\mathrm{CH}=)$; 5.46-5.38 (m, $\left.3 \mathrm{H},=\mathrm{CH}_{2}\right) ; 5.32-5.26\left(\mathrm{~m}, 3 \mathrm{H},=\mathrm{CH}_{2}\right) ; 4.66(\mathrm{~d}$, $\left.J=5.5 \mathrm{~Hz}, 2 \mathrm{H}, \mathrm{CH}_{2}\right) ; 4.62\left(\mathrm{~d}, J=5.5 \mathrm{~Hz}, 2 \mathrm{H}, \mathrm{CH}_{2}\right)$; and $4.61(\mathrm{~d}$, $\left.J=5.5 \mathrm{~Hz}, 2 \mathrm{H}, \mathrm{CH}_{2}\right) \cdot{ }^{13} \mathrm{C}-\mathrm{NMR}\left(125 \mathrm{MHz}, \mathrm{DMSO}-d_{6}\right): \delta 192.0$ $(\mathrm{C}=\mathrm{O}), 165.6,164.2,160.7,160.1,142.2,133.2,132.9,132.8$, 130.1, 127.4, 125.0, 118.4, 117.9, 117.6, 115.1, 106.4, 94.7, 92.4, $69.3\left(\mathrm{CH}_{2}\right), 68.5\left(\mathrm{CH}_{2}\right)$, and $68.2\left(\mathrm{CH}_{2}\right)$.

2.2.8. (E)-2',4,4'-Tribenzyloxy-6'-hydroxychalcone

(8). Yellow powder and insoluble in water and in methanol and soluble in acetone and in dichloromethane. Yield is
57\%. Melting point: $140-141^{\circ} \mathrm{C}$. UV $\left(\lambda_{\max } \mathrm{nm}, \mathrm{MeOH}\right)$ : 370. IR $\left(\nu \mathrm{cm}^{-1}, \mathrm{KBr}\right): 3446\left(\nu_{\mathrm{OH}}\right), 3037\left(\nu_{\mathrm{C}-\mathrm{H} \mathrm{sp} 2}\right), 2943$ $\left(v_{\mathrm{C}-\mathrm{H} \mathrm{sp} 3}\right), 2881\left(v_{\mathrm{C}-\mathrm{H} \mathrm{sp} 3}\right), 1622\left(\nu_{\mathrm{C}=\mathrm{O}}\right), 1562\left(v_{\mathrm{C}=\mathrm{C}}\right), 1421$ $\left(\nu_{\mathrm{C}=\mathrm{C}}\right)$, and $1157\left(\nu_{\mathrm{C}-\mathrm{O}}\right)$. HR-MS (ESI) $\mathrm{m} / \mathrm{z}:[\mathrm{M}+\mathrm{H}]^{+}$ calculated for $\mathrm{C}_{36} \mathrm{H}_{31} \mathrm{O}_{5}$ 543.2171; found 543.2168 . ${ }^{1} \mathrm{H}$ NMR $\left(600 \mathrm{MHz}, \mathrm{DMSO}-d_{6}\right): \delta 14.11(\mathrm{~s}, 1 \mathrm{H}, \mathrm{OH}) ; 7.68(\mathrm{~d}$, $\left.J=15.6 \mathrm{~Hz}, 1 \mathrm{H}, \mathrm{H}_{\beta}\right) ; 7.60\left(\mathrm{~d}, J=15.6 \mathrm{~Hz}, 1 \mathrm{H}, \mathrm{H}_{\alpha}\right)$; 7.56-7.54 (m, 2H, $\left.\mathrm{H}_{\mathrm{Ar}}\right) ; 7.48-7.46(\mathrm{~m}, 4 \mathrm{H}, \mathrm{HAr})$; 7.43-7.35 (m, 9H, $\left.\mathrm{H}_{\mathrm{Ar}}\right) ; 7.14\left(\mathrm{~d}, J=8.4 \mathrm{~Hz}, 2 \mathrm{H}, \mathrm{H}_{\mathrm{Ar}}\right) ; 6.90$ $\left(\mathrm{d}, J=8.4 \mathrm{~Hz}, 2 \mathrm{H}, \mathrm{H}_{\mathrm{Ar}}\right) ; 6.40\left(\mathrm{~d}, J=2.4 \mathrm{~Hz}, 1 \mathrm{H}, \mathrm{H}_{\mathrm{Ar}}\right) ; 6.24$ $\left(\mathrm{d}, J=2.4 \mathrm{~Hz}, 1 \mathrm{H}, \mathrm{H}_{\mathrm{Ar}}\right) ; 5.21\left(\mathrm{~s}, 2 \mathrm{H}, \mathrm{CH}_{2}\right) ; 5.17(\mathrm{~s}, 2 \mathrm{H}$, $\left.\mathrm{CH}_{2}\right)$; and $5.16\left(\mathrm{~s}, 2 \mathrm{H}, \mathrm{CH}_{2}\right) .{ }^{13} \mathrm{C}-\mathrm{NMR}(150 \mathrm{MHz}$, DMSO$\left.d_{6}\right): \delta 191.9(\mathrm{C}=\mathrm{O}), 166.7,164.7,161.2,160.1,142.7,136.6$, $136.2,135.7,130.1,128.9,128.7,128.5,128.4,128.1,127.9$, $127.8,127.7,127.3,124.6,115.1,105.9,94.9,92.6,71.0$ $\left(\mathrm{CH}_{2}\right), 69.7\left(\mathrm{CH}_{2}\right)$, and $69.3\left(\mathrm{CH}_{2}\right)$.

2.2.9. (E)-2',4,4',6'-Tetrabenzyloxychalcone (9). White, needle-shaped crystal insoluble in water, slightly soluble in methanol, soluble in acetone, highly soluble in dichloromethane. Yield is $67 \%$. Melting point: $139-140^{\circ} \mathrm{C}$. UV $\left(\lambda_{\max }\right.$ $\mathrm{nm}, \mathrm{MeOH}): 330$. IR $\left(\nu \mathrm{cm}^{-1}, \mathrm{KBr}\right): 3446\left(\nu_{\mathrm{OH}}\right), 3028\left(\nu_{\mathrm{C}-\mathrm{H}}\right.$ sp2 $), 2933\left(\nu_{\mathrm{C}-\mathrm{H} \mathrm{sp} 3}\right), 2864\left(\nu_{\mathrm{C}-\mathrm{H} \mathrm{sp} 3}\right), 1633\left(\nu_{\mathrm{C}=\mathrm{O}}\right), 1597$ $\left(\nu_{\mathrm{C}=\mathrm{C}}\right), 1431\left(\nu_{\mathrm{C}=\mathrm{C}}\right)$, and $1124\left(\nu_{\mathrm{C}-\mathrm{O}}\right)$. HR-MS (ESI) $\mathrm{m} / \mathrm{z}$ : $[\mathrm{M}+\mathrm{H}]^{+}$calculated for $\mathrm{C}_{43} \mathrm{H}_{37} \mathrm{O}_{5}$ 633.2641; found 633.2633. ${ }^{1} \mathrm{H}-\mathrm{NMR}\left(600 \mathrm{MHz}\right.$, DMSO-d $\left.{ }_{6}\right): \delta 7.61(\mathrm{~d}, J=8.4 \mathrm{~Hz}, 2 \mathrm{H}$, $\left.\mathrm{H}_{\mathrm{Ar}}\right)$; 7.46-7.45 (m, $\left.4 \mathrm{H}, \mathrm{H}_{\mathrm{Ar}}\right) ; 7.42-7.38\left(\mathrm{~m}, 4 \mathrm{H}, \mathrm{H}_{\mathrm{Ar}}\right)$; 7.37-7.33 (m, $\left.2 \mathrm{H}, \mathrm{H}_{\mathrm{Ar}}\right) ; 7.31-7.29\left(\mathrm{~m}, 4 \mathrm{H}, \mathrm{H}_{\mathrm{Ar}}\right) ; 7.24-7.20$ $\left(\mathrm{m}, 6 \mathrm{H}, \mathrm{H}_{\mathrm{Ar}}\right) ; 7.22\left(\mathrm{~d}, J=16.2 \mathrm{~Hz}, 1 \mathrm{H}, \mathrm{H}_{\beta}\right), 7.05(\mathrm{~d}, J=8.4 \mathrm{~Hz}$, $\left.2 \mathrm{H}, \mathrm{H}_{\mathrm{Ar}}\right) ; 6.92\left(\mathrm{~d}, J=16.2 \mathrm{~Hz}, 1 \mathrm{H}, \mathrm{H}_{\alpha}\right) ; 6.51\left(\mathrm{~s}, 2 \mathrm{H}, \mathrm{H}_{\mathrm{Ar}}\right) ; 5.17$ $\left(\mathrm{s}, 2 \mathrm{H}, \mathrm{CH}_{2}\right) ; 5.13\left(\mathrm{~s}, 2 \mathrm{H}, \mathrm{CH}_{2}\right)$; and $5.10\left(\mathrm{~s}, 4 \mathrm{H}, \mathrm{CH}_{2}\right) .{ }^{13} \mathrm{C}-$ NMR $\left(150 \mathrm{MHz}, \mathrm{DMSO}-d_{6}\right): \delta 192.9(\mathrm{C}=\mathrm{O}), 160.6,160.2$, $156.9,143.6,136.8,136.6,136.6,131.6,130.2,128.4,128.4$, $128.3,128.2,128.0,127.9,127.9,127.8,127.7,127.6,127.2$, $127.1,127.1,126.9,115.2,112.4,93.4,69.7\left(\mathrm{CH}_{2}\right), 69.6\left(\mathrm{CH}_{2}\right)$, $69.5\left(\mathrm{CH}_{2}\right)$, and $69.3\left(\mathrm{CH}_{2}\right)$.

All the spectral data of the synthesized compounds are provided in the Supplementary Materials (Tables S1-S9).

2.3. Acetylcholinesterase Inhibition Assay. AChE inhibitory activity was determined using the materials and methods as described earlier [16]. The initial mixture in each well consisted of phosphate buffer $\mathrm{pH} \mathrm{8,} \mathrm{sample} \mathrm{(studied}$ compounds or reference) prepared at different concentrations in dimethyl sulfoxide, and AChE enzyme solution $0.25 \mathrm{UI} / \mathrm{mL}$ (in phosphate buffer). This mixture was incubated at $25^{\circ} \mathrm{C}$ for 15 minutes, and the solutions of $2.4 \mathrm{mM}$ 5.5-dithio-bis-2-nitrobenzoic acid (reagent solution) and $2.4 \mathrm{mM}$ acetylthiocholine iodide (substrate solution) were then added. Continue incubating the mixture at $25^{\circ} \mathrm{C}$ for 24 minutes, and then, the absorbance was measured at $405 \mathrm{~nm}$. All samples were assayed in triplicate.

2.4. Molecular Docking Study. The methods and software are used in molecular docking study as described in the previous work [16]. Computational programs including Sybyl-X 2.0 
TABle 1: Predicted parameters by SwissADME.

\begin{tabular}{|c|c|}
\hline Classification & Parameters \\
\hline $\begin{array}{l}\text { Physicochemical } \\
\text { properties }\end{array}$ & $\begin{array}{l}\text { Formula, molecular weight, number of heavy atoms, number of aromatic heavy atoms, fraction Csp3, number } \\
\text { of rotatable bonds, number of H-bond acceptors, number of } \mathrm{H} \text {-bond donors, molar refractivity, and topological } \\
\text { polar surface area (TPSA) }\end{array}$ \\
\hline Lipophilicity & $\begin{array}{c}\text { Log Po/w (iLOGP), } \log \text { Po/w (XLOGP3), } \log \text { Po/w (WLOGP), } \log \text { Po/w (MLOGP), log Po/w (SILICOS-IT), and } \\
\text { consensus log Po/w }\end{array}$ \\
\hline Wate & $\log S$ (ESOL), $\log$ S (Ali), and $\log$ S (SILICOS-IT) \\
\hline Pharmacokinetics & $\begin{array}{l}\text { GI absorption, BBB permeant, P-gp substrate, CYP1A2 inhibitor, CYP2C19 inhibitor, CYP2C9 inhibitor, } \\
\text { CYP2D6 inhibitor, CYP3A4 inhibitor, and log Kp (skin permeation) }\end{array}$ \\
\hline & Lipinski, Ghose, Veber, Egan, Muegge, and bioavailability score \\
\hline Medicinal chemistry & PAINS, Brenk, lead-likeness, and synthetic accessibility \\
\hline
\end{tabular}

GI, gastrointestinal; BBB, blood-brain barrier; PAINS, pan-assay interference compounds.

TABLe 2: Predicted parameters by ProTox-II.

\begin{tabular}{|c|c|}
\hline Classification & Target \\
\hline Oral toxicity & LD50, toxicity class \\
\hline Organ toxicity & Hepato \\
\hline Toxicity end points & Carcinogenicity, immunotoxicity \\
\hline $\begin{array}{l}\text { Tox21-nuclear receptor signalling } \\
\text { pathways }\end{array}$ & $\begin{array}{l}\text { Aryl hydrocarbon receptor (AhR), androgen receptor (AR), androgen receptor ligand binding domain } \\
\text { (AR-LBD), aromatase, estrogen receptor alpha (ER), estrogen receptor ligand binding domain (ER- } \\
\text { LBD), and peroxisome proliferator-activated receptor gamma (PPAR-gamma) }\end{array}$ \\
\hline Tox21-stress response pathways & $\begin{array}{l}\text { Nuclear factor (erythroid-derived 2)-like 2/antioxidant responsive element (nrf2/ARE), heat shock } \\
\text { factor response element (HSE), mitochondrial membrane potential (MMP), phosphoprotein (tumor } \\
\text { suppressor) p53, and ATPase family AAA domain-containing protein } 5 \text { (ATAD5) }\end{array}$ \\
\hline
\end{tabular}

LD50, median lethal dose; Tox21, toxicology in the 21st century (a unique collaboration between several US federal agencies to develop new ways to rapidly test whether substances adversely affect human health).

[18], FlexX [19], and MOE 2008.10 [20] were used with default settings for ligands preparation, docking procedure, and interactions analysis, respectively. Protein complex 1W6R of AChE (acetylcholinesterase from Tetronarce californica in complex with the bound ligand, (-)-galantamine, at a resolution of $2.05 \AA$ ) was downloaded from Protein Data Bank [21] and utilized in this study. Docking protocols were validated by the method of pose selection [22], and the root mean square deviation (RMSD) value between redocked conformations and the original bound ligand in the cocrystallized complex which was $\leq 1.5 \AA$ would confirm the reliability of the binding ability prediction of new ligands.

\subsection{Prediction of Physicochemical Properties, Pharmacoki-} netics, and Toxicities. Physicochemical properties, pharmacokinetics, drug-likeness, and medicinal chemistry features of synthesized compounds and galantamine were predicted using the free online service SwissADME (http:// www.swissadme.ch/) [23]. Predicted toxicities of studied substances were obtained using ProTox-II web server (https://tox-new.charite.de/protox_II/) [24].

The parameters calculated by the two mentioned computational tools are given in Tables 1 and 2 .

\section{Results and Discussion}

3.1. Synthesis of Naringenin Derivatives. From naringenin, the reactions of etherification (with acetone as solvent and $\mathrm{K}_{2} \mathrm{CO}_{3}$ as the catalyst) were conducted (Scheme 1) to produce nine flavonoid derivatives with relatively high yields
(47-68\%). Two out of these compounds (6 and 9) were found as completely new structures (according to search results on SciFinder on March 8, 2021).

Naringenin has acidic phenolic $\mathrm{OH}$ groups which in alkaline condition (like $\mathrm{K}_{2} \mathrm{CO}_{3}$ ) are easily converted into anionic phenolate $\left(\mathrm{Ar}-\mathrm{O}^{-}\right)$with higher electron density than $\mathrm{OH}$ groups, as increased ability to participate in electrophilic substitution reactions. Anhydrous acetone is a good solvent that dissolves raw materials and formed products and limits the decomposition of unstable agents such as dimethyl sulfate and diethyl sulfate.

Flavanones can be easily converted to isomeric chalcones in alkaline (or acidic) media, provided within there is a hydroxyl substituent at position $2^{\prime}$ (or $6^{\prime}$ ) of chalcones (Scheme 2) [25]. Naringenin is a flavonoid with a chemical scaffold of flavanone, and opening ring $\mathrm{C}$ will form a chalcone derivative with $\mathrm{OH}$ groups at the $2^{\prime}$ and $6^{\prime}$ positions, respectively. In addition, the $\mathrm{OH}$ group at position 5 of the A ring of naringenin has an intramolecular hydrogen bond with $\mathrm{C}=\mathrm{O}$ at position 4 of the $\mathrm{C}$ ring, so this position is more difficult to be substituted than other positions. To etherify the $\mathrm{OH}$ group at this position, it is necessary to give more $\mathrm{K}_{2} \mathrm{CO}_{3}$ catalyst and perform the reaction at higher temperatures and for a longer time. With the above reaction conditions, naringenin is easy to open $\mathrm{C}$ ring to form chalcone derivatives. This was proven by experiments creating compounds $3,4,7,8$, and 9 .

The structures of synthetic substances were elucidated by UV, IR, HR-MS, ${ }^{1} \mathrm{H}-\mathrm{NMR}$, and ${ }^{13} \mathrm{C}-\mathrm{NMR}$ spectra. The UV spectra all showed characteristic maximum absorption peaks 
in the wavelength region of $270-295 \mathrm{~nm}$ for flavanones (1, 2,5 , and 6), and 330-390 nm for chalcones $(3,4,7,8$, and 9). The HR-MS spectra of the synthesized compounds were in agreement with the expected data with very small deviations.

All IR spectra have absorption bands at the wavenumbers corresponding to the characteristic functional groups of flavonoids. In which, the vibrations of the bonds $\mathrm{O}-\mathrm{H}, \mathrm{C}=\mathrm{O}, \mathrm{C}=\mathrm{C}, \mathrm{C}-\mathrm{O}, \mathrm{C}-\mathrm{H}$ are typical for the studied derivatives. The $\mathrm{OH}$ group has a signal at the wavenumber of $3400-3600 \mathrm{~cm}^{-1}$. Since the $\mathrm{C}-\mathrm{H}_{\mathrm{sp} 2}$ bond is stronger than that in $\mathrm{C}-\mathrm{H}_{\mathrm{sp} 3}$, this group $\left(\mathrm{CH}_{\mathrm{sp} 2}\right)$ stretches at higher frequencies. On the IR spectra, there appears one or more peaks located in the $3000 \mathrm{~cm}^{-1}$ region, which is typical for $\mathrm{C}-\mathrm{H}_{\mathrm{sp} 2}$ bond. The signals of $\mathrm{C}-\mathrm{H}_{\mathrm{sp} 3}$ give peaks in the region near $2900 \mathrm{~cm}^{-1}$. The $\mathrm{C}=\mathrm{O}_{\text {ketone }}$ group in ring $\mathrm{C}$ is among the easily recognizable functional groups in the IR spectra, and this group undergoes vibrational excitation at the wavenumber of $1600-1700 \mathrm{~cm}^{-1}$. A strong absorption band is also observed between 1450 and $1600 \mathrm{~cm}^{-1}$ that characterizes the double bonds of the aromatic ring.

Regarding thermodynamic equilibrium between flavanones and chalcones (Scheme 2), the spectral signals with chemical shifts of approximately $\delta 5.49 \mathrm{ppm}(\mathrm{dd}, J==12.6$; $3.0 \mathrm{~Hz}, 1 \mathrm{H}, \mathrm{O}-\mathrm{CH}<), \delta 3.32 \mathrm{ppm}(\mathrm{dd}, \mathrm{J}=17.4 ; 12.6 \mathrm{~Hz}, 1 \mathrm{H}$, $\mathrm{CH} 2), \delta 2.72 \mathrm{ppm}\left(\mathrm{dd}, J=17.4 ; 3.0 \mathrm{~Hz}, 1 \mathrm{H}, \mathrm{CH}_{2}\right)$ in ${ }^{1} \mathrm{H}-\mathrm{NMR}$ spectra, and $\delta 196.9 \mathrm{ppm}(\mathrm{C}=\mathrm{O})$ in ${ }^{13} \mathrm{C}$-NMR spectra indicate that obtained derivatives have a flavanone scaffold (compound $\mathbf{1}$ and similarly for $\mathbf{2 , 5}$, and 6) [26]. The spectral signals with chemical shifts of approximately $\delta 7.78 \mathrm{ppm}(\mathrm{d}, J$ $\left.=15.5 \mathrm{~Hz}, 1 \mathrm{H}, \mathrm{H}_{\beta}\right)$ and $\delta 7.63\left(\mathrm{~d}, J=15.5 \mathrm{~Hz}, 1 \mathrm{H}, \mathrm{H}_{\alpha}\right)$ confirm the chalcone derivatives (compound $\mathbf{4}$ and similarly for 3,7 , 8, and 9) [27]. At the same time, with the $J$ value of $\mathrm{H} \alpha \& \beta$ is approximately $16.0 \mathrm{~Hz}$, these chalcone derivatives all have the $E$ configuration.

In the ${ }^{1} \mathrm{H}-\mathrm{NMR}$ spectra of the flavanone derivatives, in addition to the signal peaks typical for this scaffold mentioned above, there are also characteristic peaks for each substance. Specifically, in monoetherified compounds, there are two singlet signals of two $\mathrm{OH}$ protons in the low magnetic field region, in which $\mathrm{OH}$ at $\mathrm{C}_{5}$ forms an intramolecular hydrogen bond with the $\mathrm{C}=\mathrm{O}_{\text {ketone }}$ group of the $\mathrm{C}$ ring, reducing the electron density around this proton, so the signal of this proton will be in a lower magnetic field region than the $\mathrm{OH}$ proton at $\mathrm{C}_{4^{\prime}}$. Substances with 2 etherified $\mathrm{OH}$ groups give a singlet signal in the low magnetic field region, while for fully etherified derivatives, this signal no longer appears on the spectra.

The ${ }^{13} \mathrm{C}$-NMR spectra of the derivatives all give characteristic peaks of $\mathrm{C}=\mathrm{O}_{\text {ketone, }}$, aromatic rings, or substituents. Due to the symmetry in the $\mathrm{B}$ ring $\left(\mathrm{C}_{2^{\prime}}\right.$ vs. $\mathrm{C}_{6^{\prime}}$ and $\mathrm{C}_{3^{\prime}}$ vs. $\mathrm{C}_{5^{\prime}}$ in flavanones and $\mathrm{C}_{2}$ vs. $\mathrm{C}_{6}$ and $\mathrm{C}_{3}$ vs. $\mathrm{C}_{5}$ in chalcones), there is an overlap signal between these carbon peaks. Therefore, there were four peaks for six aromatic carbons (ring B) on the spectra. Among them, a peak with the smallest intensity is that of carbon $\mathrm{C}_{1}$, (or $\mathrm{C}_{1}$ ) because this carbon has no attached hydrogen. Two stronger signals are for $\mathrm{C}_{2^{\prime}}$ and $\mathrm{C}_{6^{\prime}}$ (or $\mathrm{C}_{2}$ and $\mathrm{C}_{6}$ ) and $\mathrm{C}_{3^{\prime}}$ and $\mathrm{C}_{5^{\prime}}$ (or $\mathrm{C}_{3}$ and $\mathrm{C}_{5}$ ). The medium intensity peak is for $\mathrm{C}_{4^{\prime}}\left(\right.$ or $\left.\mathrm{C}_{4}\right)$.
TABLE 3: AChE inhibitory activities of flavonoid derivatives.

\begin{tabular}{lcc}
\hline No. & Compound & IC50 $(\mu \mathrm{M})$ \\
\hline 1 & $\mathbf{1}$ & $>>100.0$ \\
2 & $\mathbf{2}$ & $75.0 \pm 4.8$ \\
3 & $\mathbf{3}$ & $>>100.0$ \\
4 & $\mathbf{4}$ & $39.8 \pm 4.4$ \\
5 & $\mathbf{5}$ & $48.4 \pm 2.9$ \\
6 & $\mathbf{6}$ & $>>100.0$ \\
7 & 7 & $13.0 \pm 1.9$ \\
8 & $\mathbf{8}$ & $>>100.0$ \\
9 & $\mathbf{9}$ & $>>100.0$ \\
10 & Naringenin & $149.6 \pm 4.6$ \\
11 & Galantamine & $1.3 \pm 0.2$ \\
\hline
\end{tabular}

3.2. In Vitro Assay for Acetylcholinesterase Inhibition. Bioactivity assays were conducted on eleven samples, including nine synthetic derivatives, naringenin, and galantamine as a positive control. The results are given in Table 3 .

The results showed that all studied flavonoids had weaker AChE inhibitory activities than that of galantamine. Two new substances (6 and 9) had negligible biological effects. Four substances have improved activities over naringenin. These substances are 2, 4, 5, and 7 , which all have $\mathrm{IC}_{50}$ under $100 \mu \mathrm{M}$. Compound 7 had the best inhibitory activity against $\mathrm{AChE}$ in the synthesized flavonoids with the $\mathrm{IC}_{50}$ value of $13.0 \pm 1.9 \mu \mathrm{M}$. The two most active substances (4 and 7) were found to be chalcones derived from flavanones by ring opening and with the $\mathrm{OH}$ group at $\mathrm{C}_{5}$ position not being etherified.

3.3. Molecular Docking. The results of the molecular docking study of four compounds with the highest biological activities (compounds $\mathbf{2 , 4}, \mathbf{5}$, and $\mathbf{7}$ ) and naringenin on $\mathrm{AChE}$ are given in Table 4, Figures 1 and 2, and in the Supplementary Materials (Figures S1-S4).

The results indicated that studied substances were docked into the binding pocket of AChE with the same direction as the cocrystallized ligand (galantamine) in the protein complex. Derivatives 2, 5, and $\mathbf{7}$ and naringenin all interacted with Glu199 and Ser200 at varying degrees, and these interactions were weaker than those produced by galantamine (hydrogen bonds with Glu199 (score: 63\%, length $2.65 \AA$ ) and with Ser200 (score: $53 \%$, length: $2.80 \AA$ )). This may explain why the AChE inhibitory activities of these derivatives were weaker than that of galantamine. Substance 2 formed hydrogen bonds with both Glu199 (score: 25\%, length $2.02 \AA$ ) and Ser200 (score: $19 \%$, length: $3.01 \AA$ ); while derivatives $\mathbf{5}$ and $\mathbf{7}$ only made hydrogen bonds with Ser200 (compound 5: score: 11\%, length $3.02 \AA$; score: $16 \%$, length: $2.87 \AA$; compound 7: score: $31 \%$, length $2.90 \AA$ ) and van der Waals interaction with Glu199; meanwhile, AChE inhibitory activities were in order $2<5<7$. This may suggest that a strong interaction with Ser200 is crucial for the inhibition of AChE. Substance 4 only made a weak interaction with Ser200 (van der Waals interaction) but had a stronger biological activity than derivatives 2 and $\mathbf{5}$. This can be explained by the fact that derivative $\mathbf{4}$ had strong interactions with amino acid residues in the periphery region, 
TABLE 4: Molecular docking result of synthesized flavonoid derivatives on AChE (PDB ID: 1W6R).

\begin{tabular}{|c|c|c|c|}
\hline No. & Compound & $\begin{array}{l}\text { Docking score } \\
\left(\mathrm{kJ} \cdot \mathrm{mol}^{-1}\right)\end{array}$ & Interactions \\
\hline 2 & 2 & -21.87 & $\begin{array}{l}\text { Hydrogen bonds with Glu199 (score: } 25 \% \text {, length } 2.02 \AA \text { ) and Ser200 (score: 19\%, length: } 3.01 \AA \text { ). } \\
\text { Van der Waals interaction with Trp84, Gly119, Tyr121, Phe290, Phe330, Phe331, Tyr334, His440, } \\
\text { Gly441, and Tyr442. }\end{array}$ \\
\hline 4 & 4 & -22.40 & $\begin{array}{l}\text { Hydrogen bonds with Tyr70 (score: } 56 \% \text {, length: } 2.05 \AA \text { ) and Tyr121 (score: 69\%, length } 2.54 \AA \text { ). } \\
\text { Van der Waals interaction with Asp72, Trp84, Gly118, Gly119, Ser200, Trp233, Trp279, Phe288, } \\
\text { Phe290, Phe330, Phe331, Tyr334, and His440 }\end{array}$ \\
\hline 5 & 5 & -18.86 & $\begin{array}{c}\text { Hydrogen bonds with Trp84 (score: } 33 \% \text {, length } 1.80 \AA \text { ), Tyr121 (score: } 82 \% \text {, length: } 2.58 \AA \text { ), and } \\
\text { Ser200 (score: } 11 \% \text {, length } 3.02 \AA \text {; score: } 16 \% \text {, length: } 2.87 \AA \text { ). } \\
\text { Van der Waals interaction with Gln69, Gly117, Ser122, Gly123, Gly118, Gly119, Glu199, Phe290, } \\
\text { Phe330, Phe331, and His440. }\end{array}$ \\
\hline 7 & 7 & -13.30 & $\begin{array}{c}\text { Hydrogen bonds with Ser200 (score: 31\%, length } 2.90 \AA \text { ). } \\
\text { Van der Waals interaction with Asp72, Trp84, Gly117, Gly118, Gly119, Tyr121, Tyr130, Glu199, } \\
\text { Trp279, Phe290, Phe330, Phe331, Tyr334, His440, Gly441, and Ile444. }\end{array}$ \\
\hline 10 & Naringenin & -21.12 & $\begin{array}{c}\text { Hydrogen bonds with His440 (score: } 20 \% \text {, length } 2.00 \AA \text { ). } \\
\text { Van der Waals interaction with Asp72, Trp84, Asn85, Gly118, Gly119, Tyr121, Ser122, Glu199, } \\
\text { Ser200, Phe330, Gly441, and Tyr442. }\end{array}$ \\
\hline
\end{tabular}

Asn, asparagine; Asp, aspartate; Gln, glutamine; Glu, glutamate; Gly, glycine; His, histidine; Ile, isoleucine; Phe, phenylalanine; Ser, serine; Trp, tryptophan; Tyr, tyrosine.

which were hydrogen bonds with Tyr70 (score: $56 \%$, length: $2.05 \AA$ ) and Tyr121 (score: 69\%, length $2.54 \AA$ ). This also suggests an important role of peripheral interaction for the enzyme inhibitory activities of the compounds. Substance 7 made van der Waals interactions with 4/5 amino acids of the peripheral region (Asp72, Tyr121, Trp279, and Phe331); this contribution also explained partially the highest biological activity of derivative 7 in the studied compounds. Derivatives 2, 4, 5, and 7, naringenin, and galantamine all interacted with His440 (an amino acid of the catalytic triad). In addition to hydrogen bonding with His440 (score: 20\%, length $2.00 \AA$ ) , naringenin could only make van der Waals interactions with two amino acids Glu199 and Ser200 and with two amino acids Asp72 and Tyr121 of the peripheral region. This may partially explain why the AChE inhibitory activity of naringenin is weaker than that of derivatives 2,4 , 5 , and 7 .

\subsection{Prediction Physicochemical Properties, Pharmacokinetics,} and Toxicities. Predicted physicochemical properties, pharmacokinetics, and toxicities of synthesized compounds and galantamine are reported in the Supplementary Materials (Tables S10 and S11). The results showed that compound 7 (the most potential AChE inhibitor in this study) was predicted to have high capacities of absorption from the gastrointestinal tract and crossing the blood-brain barrier. This derivative was also expected to inhibit CYP1A2, CYP2C9, and CYP3A4. Regarding drug-likeness, derivative
7 satisfied the filters of Lipinski, Ghose, and Egan. This substance also did not have any alert on pan-assay interference compounds (PAINS).

In terms of toxicity, compound 7 was predicted to have a low acute oral toxicity with LD50 of $3800 \mathrm{mg} / \mathrm{kg}$ (toxicity class of 5), which was much less toxic than galantamine (LD50 of $85 \mathrm{mg} / \mathrm{kg}$ and toxicity class of 3 ). The compound was expected with high probabilities (0.71-0.98) and not to have hepatotoxicity, mutagenicity, and cytotoxicity or not to be active on aryl hydrocarbon receptor, androgen receptor ligand binding domain, aromatase, estrogen receptor ligand binding domain, peroxisome proliferator-activated receptor gamma, nuclear factor (erythroid-derived 2)-like 2/antioxidant responsive element, heat shock factor response element, and ATPase family AAA domain-containing protein 5. The compound was also assumed not to have carcinogenicity or not to be active on mitochondrial membrane potential, phosphoprotein (tumor suppressor) p53 (probabilities of $0.50-0.68$ ).

It was anticipated that substance 7 had immunotoxicity with a high probability (0.92). In addition, this derivative was also expected to be active on estrogen receptor alpha, but with a correspondingly low probability of 0.59 .

Thus, in addition to having the most potential AChE inhibitory activity among synthetic derivatives, compound 7 was also expected to have good pharmacokinetic properties, drug-likeness, and low toxicities on most common targets. Therefore, this is a promising substance for further studies. 

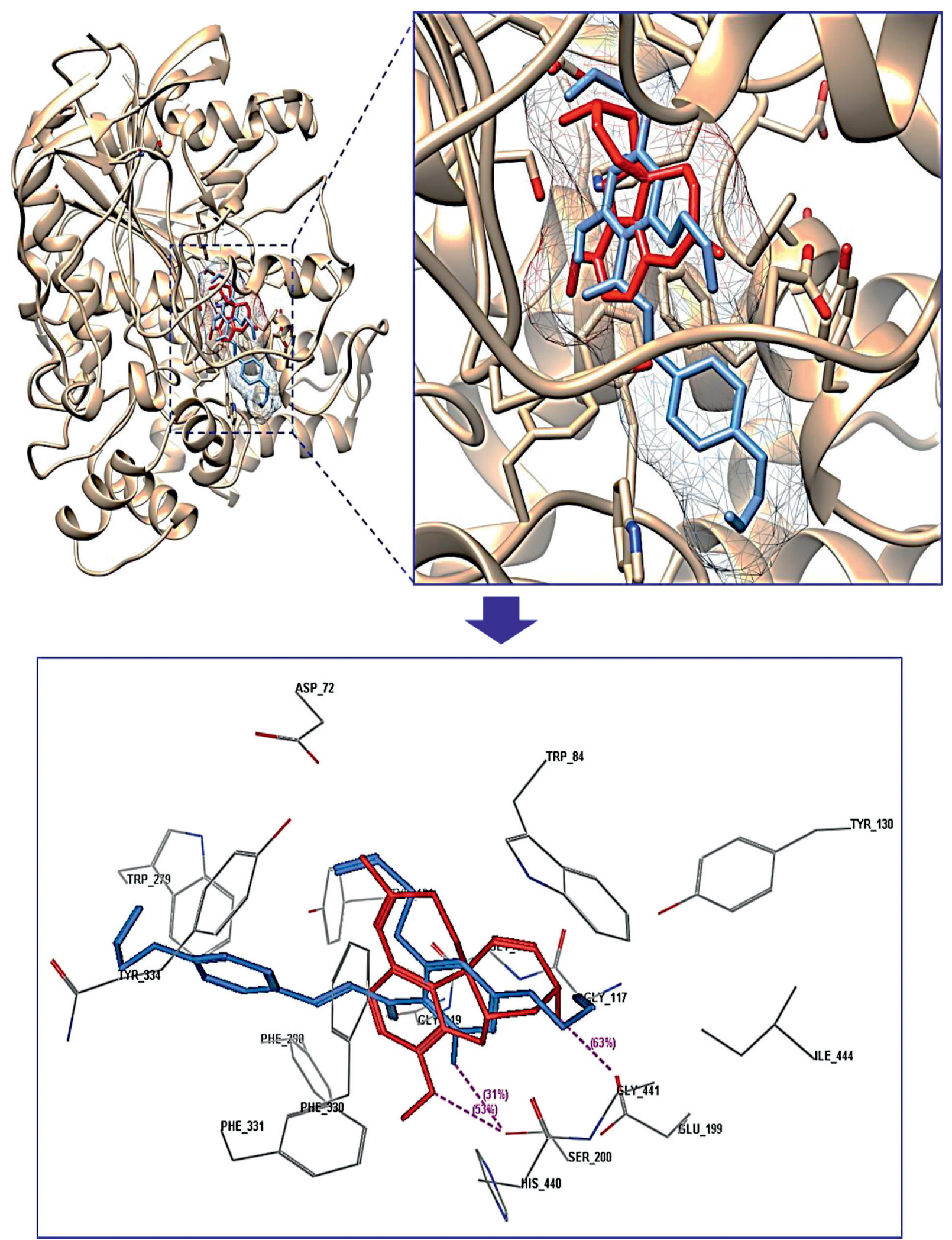

FIGURE 1: Alignment of docked pose of compound 7 with galantamine as cocrystallized ligand in the AChE complex (PDB ID: 1W6R). Compound 7 is rendered in cornflower blue, and galantamine is rendered in red. Asp, aspartate; Trp, tryptophan; Gly, glycine; Tyr, tyrosine; Glu, glutamate; Ser, serine; Phe, phenylalanine; His, histidine; Ile, isoleucine. 


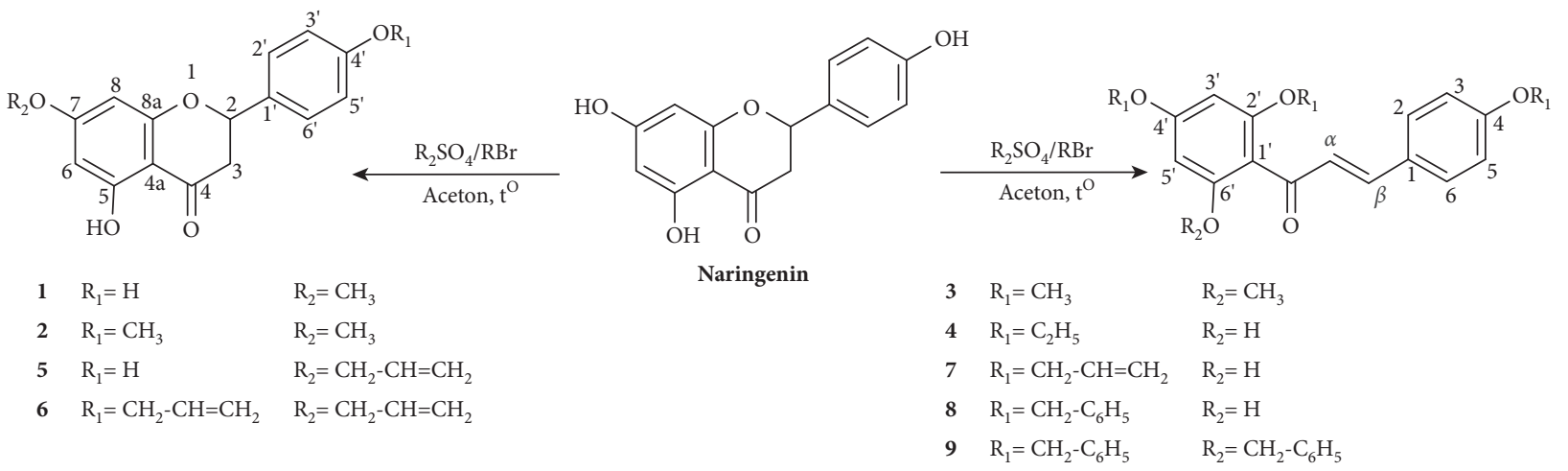

Scheme 1: Synthesis of flavonoid derivatives from naringenin.

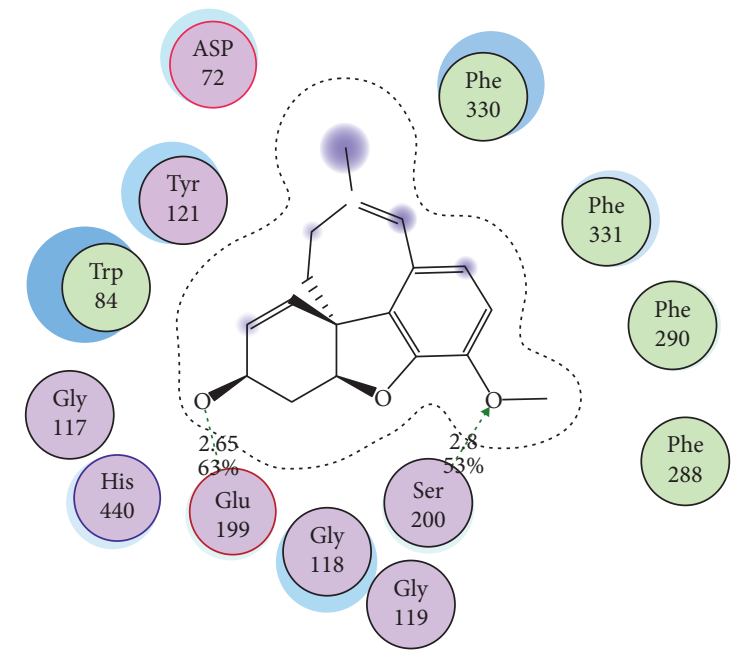

Galantamine

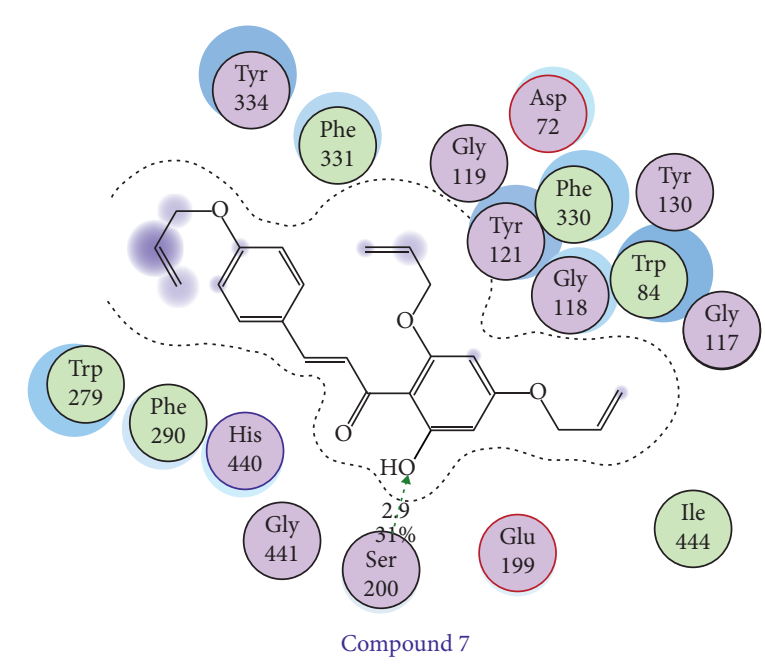

$\bigcirc \begin{aligned} & \text { solvent residue } \\ & \text { metal complex }\end{aligned}$
$\ldots .$. solvent contact
$\ldots .$. metal contact
$\bigcirc \begin{aligned} & \text { receptor } \\ & \text { exposure }\end{aligned}$
(O)

[0]+ arene-cation$$
\text { exposure }
$$

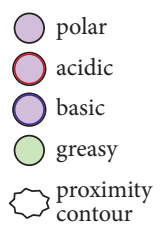

$$
\begin{aligned}
& \ldots . . \text { sidechain acceptor } \\
& \ldots \ldots \text { sidechain donor } \\
& \ldots . . \\
& \ldots \text { backbone acceptor } \\
& \\
& \quad \text { backbone donor } \\
& \text { exposure }
\end{aligned}
$$

Figure 2: Mode of interaction of galantamine and compound 7 with the enzyme AChE (depicted in two-dimensional format). Asp, aspartate; Trp, tryptophan; Gly, glycine; Tyr, tyrosine; Glu, glutamate; Ser, serine; Phe, phenylalanine; His, histidine; Ile, isoleucine.

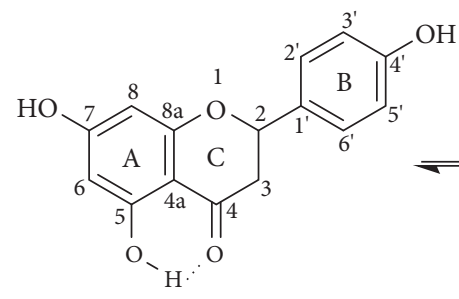

Naringenin-Flavanone<smiles>O=C(/C=C/c1ccc(O)cc1)c1c(O)cc(O)cc1O</smiles>

Naringenin-Chalcone

Scheme 2: The conversion between flavanone and chalcone. 


\section{Conclusions}

In this study, based on the Williamson method, nine flavonoid derivatives, including two completely new structures, were synthesized from naringenin. The AChE enzyme inhibitory activities of the compounds were determined. The results indicated that four substances with improved activities compared to naringenin were obtained, in which compound 7 was the strongest enzyme inhibitor in the synthesized derivatives $\left(\mathrm{IC}_{50} 13.0 \pm 1.9 \mu \mathrm{M}\right.$ ) with promising predicted profile in physicochemical properties, pharmacokinetics, and toxicities. Two new substances had negligible biological activities. However, they should be used in further studies on other targets. The molecular docking revealed that strong interactions with amino acid residue Ser200 of the catalytic triad and those of the peripheral region are crucial for strong inhibitory activities against AChE.

\section{Data Availability}

The data used to support the findings of this study are included within the article.

\section{Conflicts of Interest}

The authors declare that they have no conflicts of interest.

\section{Acknowledgments}

This work was supported by University of Medicine and Pharmacy, Hue University (Grant number: 17/20 for TheHuan Tran).

\section{Supplementary Materials}

Tables S1-S9: spectral data of compounds 1-9. Figure S1: mode of ligand interaction of compound 2 with AChE. Figure S2: mode of ligand interaction of compound 4 with AChE. Figure S3: mode of ligand interaction of compound 5 with AChE. Figure S4: mode of ligand interaction of naringenin with AChE. Table S10: pharmacokinetics, druglikeness, and medicinal chemistry evaluation of studied compounds by SwissADME online service. Table S11: predicted toxicities of studied compounds by ProTox-II web server. (Supplementary Materials)

\section{References}

[1] C. L. Masters, R. Bateman, K. Blennow, C. C. Rowe, R. A. Sperling, and J. L. Cummings, "Alzheimer's disease," Nature Reviews Disease Primers, vol. 1, no. 1, Article ID 15056, 2015.

[2] J. Lalut, H. Payan, A. Davis et al., "Rational design of novel benzisoxazole derivatives with acetylcholinesterase inhibitory and serotoninergic 5-HT4 receptors activities for the treatment of Alzheimer's disease," Scientific Reports, vol. 10, no. 1, p. $3014,2020$.

[3] J. Jia, C. Wei, S. Chen et al., "The cost of Alzheimer's disease in China and re-estimation of costs worldwide," Alzheimer's and Dementia, vol. 14, no. 4, pp. 483-491, 2018.
[4] H. Hampel, M.-M. Mesulam, A. C. Cuello et al., "The cholinergic system in the pathophysiology and treatment of Alzheimer's disease," Brain, vol. 141, no. 7, pp. 1917-1933, 2018.

[5] M. Son, C. Park, S. Rampogu, A. Zeb, and K. Lee, "Discovery of novel acetylcholinesterase inhibitors as potential candidates for the treatment of Alzheimer's disease," International Journal of Molecular Sciences, vol. 20, no. 4, p. 1000, 2019.

[6] A. Fais, A. Kumar, R. Medda et al., "Synthesis, molecular docking and cholinesterase inhibitory activity of hydroxylated 2-phenylbenzofuran derivatives," Bioorganic Chemistry, vol. 84, pp. 302-308, 2019.

[7] G. L. Delogu, M. J. Matos, M. Fanti et al., "2-phenylbenzofuran derivatives as butyrylcholinesterase inhibitors: synthesis, biological activity and molecular modeling," Bioorganic \& Medicinal Chemistry Letters, vol. 26, no. 9, pp. 2308-2313, 2016.

[8] J. Zhang and C.-S. Jiang, "Synthesis and evaluation of coumarin/ piperazine hybrids as acetylcholinesterase inhibitors," Medicinal Chemistry Research, vol. 27, no. 6, pp. 1717-1727, 2018.

[9] M. S. Uddin, M. T. Kabir, K. Niaz et al., "Molecular insight into the therapeutic promise of flavonoids against alzheimer's disease," Molecules, vol. 25, no. 6, p. 1267, 2020.

[10] H. Soltana, M. De Rosso, H. Lazreg, A. D. Vedova, M. Hammami, and R. Flamini, "LC-QTOF characterization of non-anthocyanic flavonoids in four Tunisian fig varieties," Journal of Mass Spectrometry, vol. 53, no. 9, pp. 817-823, 2018.

[11] M. Denaro, A. Smeriglio, and D. Trombetta, "Antioxidant and anti-inflammatory activity of citrus flavanones mix and its stability after in vitro simulated digestion," Antioxidants, vol. 10, no. 2, p. 140, 2021.

[12] F. A. Pinho-Ribeiro, A. C. Zarpelon, V. Fattori et al., "Naringenin reduces inflammatory pain in mice," Neuropharmacology, vol. 105, pp. 508-519, 2016.

[13] A. Ahmadi, P. Hassandarvish, R. Lani et al., "Inhibition of chikungunya virus replication by hesperetin and naringenin," RSC Advances, vol. 6, no. 73, pp. 69421-69430, 2016.

[14] D. Arul and P. Subramanian, "Naringenin (Citrus flavonone) induces growth inhibition, cell cycle arrest and apoptosis in human hepatocellular carcinoma cells," Pathology and Oncology Research, vol. 19, no. 4, pp. 763-770, 2013.

[15] S. Ghofrani, M.-T. Joghataei, S. Mohseni et al., "Naringenin improves learning and memory in an Alzheimer's disease rat model: insights into the underlying mechanisms," European Journal of Pharmacology, vol. 764, pp. 195-201, 2015.

[16] T.-S. Tran, T.-D. Tran, T.-H. Tran et al., "Synthesis, in silico and in vitro evaluation of some flavone derivatives for acetylcholinesterase and BACE-1 inhibitory activity," Molecules, vol. 25 , no. 18, p. 4064, 2020.

[17] J. Kozłowska, E. Grela, D. Baczyńska, A. Grabowiecka, and M. Anioł, "Novel O-alkyl derivatives of naringenin and their oximes with antimicrobial and anticancer activity," Molecules (Basel, Switzerland), vol. 24, no. 4, p. 679, 2019.

[18] Sybyl X. 2.0, 2021, https://sybyl-x.software.informer.com/2.0/.

[19] LeadIT 2.0.2, 2021, https://www.biosolveit.de/LeadIT/.

[20] S.S.W. MOE, 2008, 10 edition, Chemical Computing Group Inc., Suite 910, Montreal, Quebec, Canada H3A 2R7, 2021, https://www.chemcomp.com/.

[21] Protein Data Bank, 2021, https://www.rcsb.org/structure/ 1W6R.

[22] K. E. Hevener, W. Zhao, D. M. Ball et al., "Validation of molecular docking programs for virtual screening against dihydropteroate synthase," Journal of Chemical Information and Modeling, vol. 49, no. 2, pp. 444-460, 2009. 
[23] A. Daina, O. Michielin, and V. Zoete, "SwissADME: a free web tool to evaluate pharmacokinetics, drug-likeness and medicinal chemistry friendliness of small molecules," Scientific Reports, vol. 7, no. 1, p. 42717, 2017.

[24] P. Banerjee, A. O. Eckert, A. K. Schrey, and R. Preissner, "ProTox-II: a webserver for the prediction of toxicity of chemicals," Nucleic Acids Research, vol. 46, no. W1, pp. W257-W263, 2018.

[25] M. Ninomiya and M. Koketsu, "Minor flavonoids (chalcones, flavanones, dihydrochalcones, and aurones)," in Natural Products: Phytochemistry, Botany and Metabolism of Alkaloids, Phenolics and Terpenes, K. G. Ramawat and J.-M. Mérillon, Eds., Springer, Berlin, Heidelberg, pp. 1867-1900, 2013

[26] J. Kozłowska, B. Potaniec, B. Żarowska, and M. Anioł, "Synthesis and biological activity of novel O-alkyl derivatives of naringenin and their oximes," Molecules (Basel, Switzerland), vol. 22, no. 9, p. 1485, 2017.

[27] T. Yamamoto, M. Yoshimura, F. Yamaguchi et al., "Antiallergic activity of naringenin chalcone from a tomato skin extract," Bioscience, Biotechnology, and Biochemistry, vol. 68, no. 8, pp. 1706-1711, 2004. 